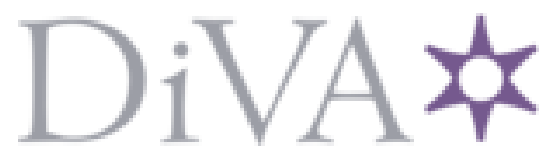

http://www.diva-portal.org

This is the published version of a paper presented at Target and Background Signatures, 23-24 September 2015, Toulouse, France.

Citation for the original published paper:

Andersson, K., Kariis, H., Hult, G. (2015)

A systems approach to stealth on the ground revisited.

In: Karin U. Stein \& Ric H. M. A. Schleijpen (ed.), Target and Background Signatures SPIE International Society for Optical Engineering

SPIE Proceedings

https://doi.org/10.1117/12.2194844

N.B. When citing this work, cite the original published paper.

Copyright: 2015 Society of Photo Optical Instrumentation Engineers. One print or electronic copy may be made for personal use only. Systematic reproduction and distribution, duplication of any material in this paper for a fee or for commercial purposes, or modification of the content of the paper are prohibited.

Permanent link to this version:

http://urn.kb.se/resolve?urn=urn:nbn:se:fhs:diva-5645 


\title{
A Systems Approach to Stealth on the Ground Revisited
}

\author{
Kent E Andersson ${ }^{* a}$, Hans Kariis ${ }^{\mathrm{b}}$, Gunnar Hult ${ }^{\mathrm{a}}$ \\ ${ }^{a}$ Swedish Defence University, Box 278 05, SE-115 93 Stockholm, Sweden \\ bwedish Defence Research Agency, SE-581 11 Linköping, Sweden
}

\begin{abstract}
This new security development is expected to increase interest from Northern European states in supporting the development of conceptually new stealthy ground platforms, incorporating a decade of advances in technology and experiences from stealth platforms at sea and in the air. The scope of this case study is to draw experience from where we left off. At the end of the 1990s there was growing interest in stealth for combat vehicles in Sweden. An ambitious technology demonstrator project was launched. One of the outcomes was a proposed Systems Engineering process tailored for signature management presented to SPIE in 2002.(Olsson et.al, A systems approach..., Proc. SPIE 4718 ) The process was used for the Swedish/BAE Systems Hägglunds AB development of a multirole armored platform (The Swedish acronym is SEP). Before development was completed there was a change of procurement policy in Sweden from domestic development towards Governmental Off-The-Shelf, preceded by a Swedish Armed Forces change of focus from national defense only, towards expeditionary missions. Lessons learned, of value for future development, are presented. They are deduced from interviews of key-personnel, on the procurer and industry sides respectively, and from document reviews.
\end{abstract}

Keywords: Stealth, Low observable technology, Signature Management, Camouflage, Military Utility, MilitaryTechnology, Systems Engineering, Combat vehicle, SEP

\section{INTRODUCTION}

The worsening security environment in Northern and Eastern Europe increases the need to give priority to national defense tasks. This in turn increases interest in high-end technology. The procurement of new army materiel during the first decade of this century has been characterized by states purchasing off-the-shelf products, and thereby sponsoring only incremental development. The focus of armed forces has been on force protection in asymmetric warfare. Hence, although there has been great development in sensor technology, thereby increasing the potential threat, and in materials science, thereby increasing the possibilities to reduce signatures, the interest in signature management and camouflage for combat vehicles has been modest. However, the emergence of highly capable and possibly adversarial systems in the vicinity of Northern and Eastern Europe highlights the need to look at new concepts for low-signature land platforms, partly by leveraging results from low-signature naval and air systems .

Up until the end of the 'Cold war' Swedish doctrine was tuned to national defense. Significant competence and experience in signature management for combat vehicles was gathered in development projects like the S-tank (Strv 103) and the $\mathrm{CV}_{90}{ }^{\dagger}$ and also in tests and trials in connection with procuring the Leopard main battle tank (Lindström, 2015). Then in 1996 a joint service program, 'SAT/Mark’ was initiated and was managed within the Swedish Defence Materiel

\footnotetext{
* kent.andersson@fhs.se; phone 46855342836 ; fax 46855342598

† Combat Vehicle 90 is a family of Swedish tracked combat vehicles designed by FMV, Hägglunds and Bofors during the mid-1980s and early 1990s (Wikipedia)

* Swedish for Low Observable Technology/Ground
} 
Agency's (FMV) R\&D organization. It was an integrated effort to consolidate knowledge in low observable technology (LOT) from all services. The initiative was supported by the Swedish Armed Forces (SwAF), the Swedish Defence Research Agency (FOI) and industry representatives. The program included establishing competence networks, enhancing design and system integration skills, developing joint defense standards and coordinating national resources in the signature field. In two technology demonstrator projects the signature management concepts and low observable technologies were tested in practice. The second SAT/Mark demonstrator (See Figure 1) was developed with signature requirements influencing design already from basic construction and was developed by four companies as a joint project. Hägglunds Vehicle AB had responsibility for the chassis, electro-optic sights and environmental situational awareness. Saab Barracuda AB developed the signature reduction coating and an inflatable camouflage system. Bofors Defence AB was responsible for the turret and the weapon system and Saab Tech Systems AB was responsible for the sensor systems. The experiences of both FMV and industry from the development of the SAT/Mark demonstrator also resulted in a proposed 'Systems approach' to developing stealth on the ground, presented to SPIE in $2002^{1}$.

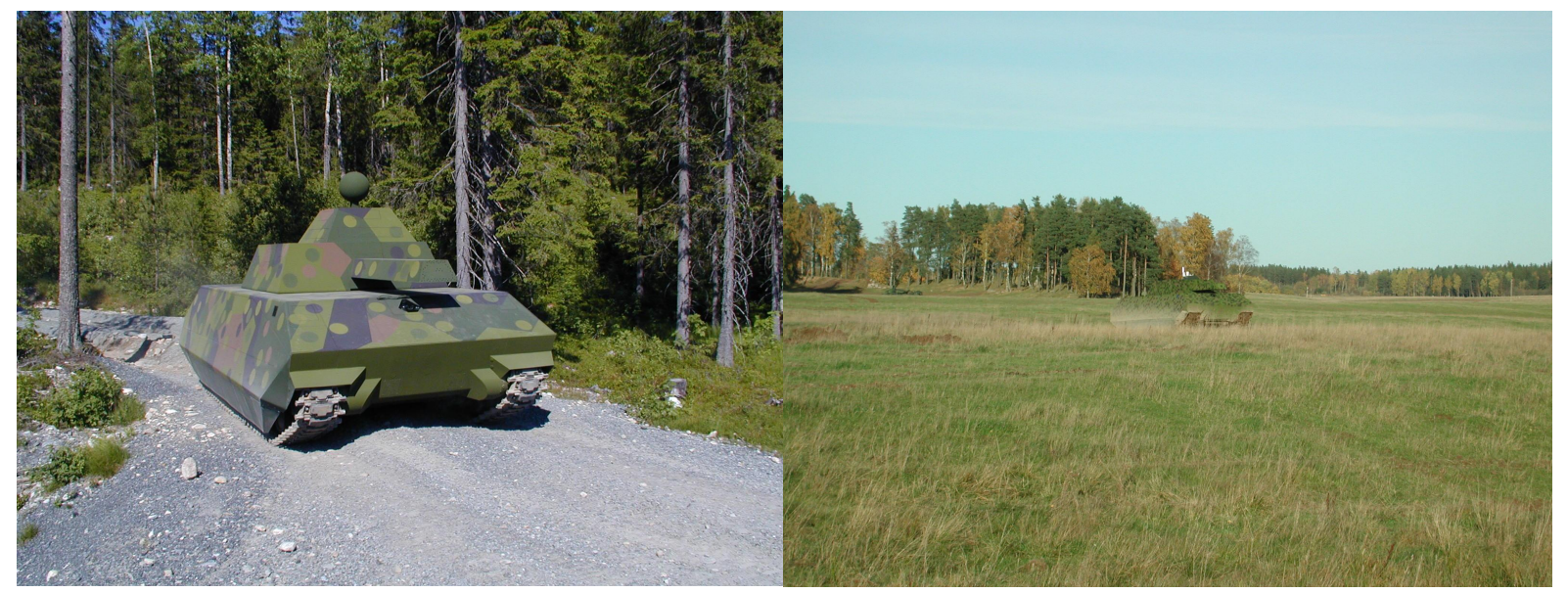

Figure 1. The second low observable technology demonstrator. On the left the characteristic low radar signature shape of the vehicle is prominent. The picture on the right demonstrates the visual signature at a more tactically relevant distance. Photos courtesy of BAE Hägglunds AB and of Saab Barracuda AB respectively.

For the first time since then, the Systems approach has been implemented in the 'SEP' program. SEP is a Swedish abbreviation for Multirole Armored Platform. The FMV project started in 1994 as a pre-study and was terminated in 2008 without entering series production. The reason for the project was a need identified to replace 7-8000 vehicles in the Swedish Armed Forces (SwAF) during the period 2005 to 2015, ranging from troop transporters to combat vehicles in mechanized units. The SEP concept meant pursuing a solution to replace all these different types of vehicles with a modular concept designed to meet a total of 24 different roles. One of the fundamental design requirements was exchangeable wheeled and tracked chassis. The development originated from an idea that "new technology should bring improvements in cost-effectiveness and performance, initially in the following areas: net load, internal volume, flexibility in conjunction with a high degree of family relationship, signature and survivability, internal environment and system cost."2 The technologies of special interest to support these development targets were identified at an early stage as: electric transmission, continuous rubber track, decoupled running gear, composite fiber hull, add-on ballistic protection and lastly, multispectral signature adaptation, especially IR and radar. During the study and concept analysis phases there were eight test-rigs built for proof of concepts. The SAT/Mark demonstrators are seen as two of them. In 2006 Hägglunds Vehicles AB (later BAE Systems Hägglunds AB) was awarded a contract for the first phase development of SEP. See Figure 2. In the meantime there was a change of procurement strategy ${ }^{3}$ towards off-the-shelf or development only with international partners. In 2008 it became clear that the Swedish government was not going to find a partner and the project was terminated. ${ }^{4}$ 


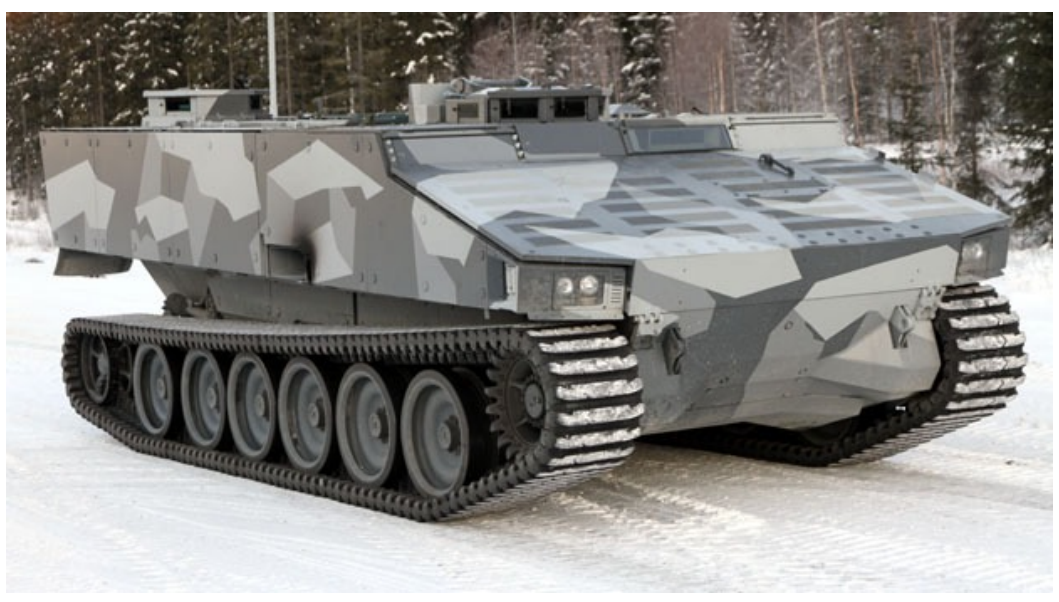

Figure 2. This is one of four pre-series SEP vehicles produced by BAE Systems Hägglunds AB in 2009. Photo courtesy of Rickard O. Lindström.

However, SEP is regarded here as a unique combat vehicle development project from a signature management point of view. There were ambitious requirements of the signature from the beginning and an ambition to balance these with requirements for other attributes, drawing on experiences from the technology demonstrator programs. It is some time since the project was terminated in 2008 , but since signature management is assessed to be of increasing importance to survivability of ground forces in future conflicts, it is important not to lose sight of the lessons identified. Hence, the scope of this paper is to collect possible new lessons and possibly to complement the systems approach to developing stealth on the ground.

In the second section of the paper the systems approach to development is presented with the aim of capturing key features and earlier lessons. In the subsequent section the interview study method is presented. In the following section results, in the form of statements from the respondents, are presented and discussed. Finally conclusions are drawn and a list of lessons is presented.

\section{THE SYSTEMS APPROACH TO STEALTH ON THE GROUND}

A literature search on survivability indicates a lot of research into aircraft and ship survivability, but considerably less on ground combat vehicles. This was also one of the main drivers behind the LOT $^{\S}$ demonstrator program, SAT/Mark, reported on. Therefore, our theoretical basis for using signature management technology to increase survivability is the work presented by Robert E. Ball on The fundamentals of aircraft combat survivability analysis and design ${ }^{5}$. Ball states that the interdependence between attributes of a platform due to survivability enhancement features has been the motivation for developing a survivability discipline in systems engineering - thereby advocating a systems approach to development. These points, emphasized as the most important in aircraft combat survivability analysis and design, can easily be transferred to systems engineering in other vehicle domains.

- "The design should be properly, not necessarily evenly, balanced..." between features reducing the probability of being killed given a hit (vulnerability), and features reducing the probability of being hit (susceptibility). "Remember the goals of survivability are to increase the cost effectiveness of the aircraft as a weapon system and to

$\S$ Low Observable Technology 
increase the likelihood the crew of an aircraft that is terminally damaged will be recovered - not to design the most survivable aircraft possible". 5 (pp174-175)

- "Survivability must be seriously considered by everyone during the early design phases of the aircraft...Retrofitting survivability features into existing aircraft, or adding them in a full-scale development phase, usually creates weight, cost, and other performance penalties that could have been avoided..., $5(\mathrm{p} 175)$

- "The people who are responsible for the survivability analysis and design of an aircraft weapon system must not work in a vacuum or be ignored. They must have contact with the aircraft designers, the program manager, and the operators on a continuing basis."

Olsson et al find that what is true for aircraft and ships is also true for ground combat vehicles - a systems approach is necessary. "Signature management involves in principle all the components in a vehicle and it is not always evident how the interactions between sub-systems add up to the total systems signature". That is to say, an object signature is a system attribute. Their work presented as a systems approach is seen here as a tailoring of the survivability discipline of systems engineering to developing stealthy ground combat vehicles. Their paper presents lessons learned in the process of developing the technology demonstrators, from a signature management perspective, particularly in terms of functional analysis, system requirement analysis and systems modeling. Hence a state of the art generic systems engineering process is a prerequisite. Their lessons have been derived from the text, formulated in bullets and used as background in the study. They are presented, with new lessons, in the conclusion section.

\section{METHOD AND LITERATURE}

The focus is on the Swedish development of the multipurpose armored vehicle SEP. The SAT/Mark program, including the two signature-management-technology demonstrator projects, is regarded here as an activity within the concept stage of the SEP development process.

Data was collected using semi-structured interviews of key personnel from the stakeholders, the procurer and the suppliers, and from reviews of relevant documents. Note that some of the respondents only took part in the SAT/Mark program. The respondents were chosen in an effort to find people in key roles during the SEP development from initiation to termination (see the list of respondents with roles in the references section).

The basic structure of the interviews was to ask respondents to describe, from their perspective, each step of the development process, and to ask, given their experiences, what could be learned for future projects. For that purpose, this stepped process was assumed to follow the Concept and Development stages in the ISO 15288 life cycle as described by INCOSE in the Systems Engineering Handbook. ${ }^{6}$ See Figure 3.

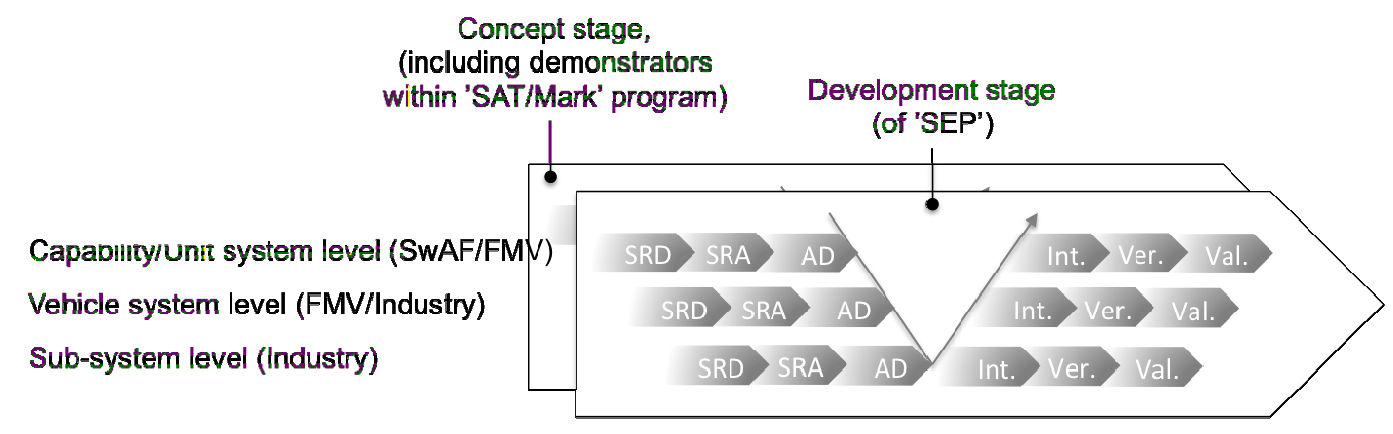

Figure 3 A model of the SEP development process for the Concept and Development consecutive life cycle stages. The technical SE sub processes are seen as steps in the process used recursively at each system level: Stakeholder Requirements Definition (SRD), System Requirements Analysis (SRA), Architectural Design (AD), Integration (Int.), Verification (Ver.) and Validation (Val.). The main actors at each system level are indicated. 
Document reviews were used, when such documents were available, to support key statements from the respondents. There are FOI reports (in Swedish) from the early stages of the SAT/Mark program, in 1998, concerning issues in modeling and requirements specification ${ }^{7,8}$, and a handbook on signature management for ground vehicles, $2003^{9}$. Most documentation from the projects is confidential. Two key study documents were, however, made available to the authors: a report on resulting 'FMV guidelines for requirements on signature management' ${ }^{10}$ and a 'Preliminary target capability document' specifying SwAF needs ${ }^{11}$. A lessons learned report from the SEP project management to SwAF is partly unclassified and useful for reviewing the schedule and key management events ${ }^{12}$.

\section{RESULTS AND DISCUSSION - NEW LESSONS?}

The semi-structured interviews were transcribed. Only those statements underpinning the discussion are outlined here.

\subsection{The stakeholder requirements definition}

"The purpose of the Stakeholder Requirements Definition Process is to define the requirements for a system that can provide the services needed by users and other stakeholders in a defined environment. "6(p54),13

In broad terms the SAT/Mark demonstrators were used to show how far it was possible to push signature management requirements, given technologies and production methods available. From measurements obtained and evaluations made in the demonstrator project, the SEP signature requirements were then derived with adjustments for considering: the mission needs, the predicted likelihood of sensor and weapon system occurrence in theaters during the SEP life cycle, and the cost of effective signature reduction in the respective sensor domains.

The requirements process that emerges from the interviews was shaped during the demonstrator programs in an atmosphere of openness and cooperation between government agencies and industries. It is described as a result of the so-called 'Swedish development model' and is promoted as a success factor by the respondents, both from the government and industry sides. It is an important factor in explaining how Sweden has been able to produce so many competitive platforms for air, navy and ground forces, one respondent explains. Sweden has become competitive through working collaboratively in integrated project teams of the agencies, SwAF and industry working together. This has allowed industry to benefit from the development and gather expertise. In practice this policy led to a sharing of systems responsibility between FMV and the suppliers. Those were the prerequisites both for the demonstrator projects and for the SEP development project, but changed due to a new strategic procurement policy in $2007^{3}$ before ordering the series vehicles. In the European Union today a similar approach is only possible for procurements of great national security interest - combat vehicles are currently not included.

The end-users were represented during the demonstrator project and hence could convey user needs continuously. Documenting needs in concepts of operations (ConOps) was considered but was decided not to be worth the extra effort, because of strained resources. There is, of course, a risk that traceability to stakeholder needs degenerates with time and that needs are not easily communicated to the whole project. It seems to have worked well here, thanks to the integrated project approach and staff continuity.

Thus, the government requirements analysis was supported by end-users, but also by scientists and industry experts working in teams during development of the customer requirements specification, thereby producing benefits from covering the entire technology readiness level scale (TRL), as pointed out by one of the respondents.

The participation of the SEP contractor in the requirements specification is especially emphasized. The benefits were highlighted by one respondent describing Hägglunds involvement. Hägglunds participated in the SEP project as early as the SwAF multirole study and in the SAT/Mark program. Therefore, the stakeholder requirements analysis for SEP was considered relatively straightforward from Hägglunds' perspective since, in principle, the requirements from the SAT/Mark project were reused and slightly modified. In the SwAF study Hägglunds contributed with an assessment of 
important technologies and at least twenty different concepts using an assessment model designed by FMV, the respondent explains. The number of concepts was reduced to about four. SwAF and FMV refined the criteria in the assessment model and one concept was later chosen. Hägglunds built a first demonstrator to support the validation of desired capabilities. They then built a second demonstrator to de-risk the design since a few of the technologies used were assessed as high risk. The system requirements were refined continuously during the process. In total, three demonstrators were built before the SEP development started in 2006, two to demonstrate function and one to de-risk technology. During this process Hägglunds were asked, the respondent explains, not only to design according to system requirements, but also to challenge them. Consequences of design requirements, e.g. those having secondary impacts on other capabilities, were continuously reported back to FMV. Key requirements were allowed to have the impact reported and others were modified, the respondent ends. The description of Hägglunds involvement illustrates how two-way communication is aided. The contractor learns to better understand the needs and the procurer side learns to better understand which requirements are pushing cost and complexity, and hence project risks. An observation is the way of working, promoted by the respondents, is completely in accordance with Ball's third point (see section 2). The net result is likely to be a more balanced requirements specification.

Initially relatively basic high-level requirements were produced by SwAF in a document no longer than one page. The requirements from SwAF were not quantified. Instead they were formulated in relation to what had been accomplished in the CV90 project. This was enough for the early studies. In 2006, in conjunction with an FMV request for a quotation for the development of SEP, SwAF issued preliminary target capability documents ${ }^{11}$ for the different roles of the vehicle. These, and the customer specification from FMV, were the only documents formally conveying SwAF capability needs to industry. In the preliminary capability target document, SwAF needs were formulated using a configuration of threat sensors with different elevations, very much like the situation depicted in Figure 1 in Olsson et al., 2002 ${ }^{1}$. The measures of performance and situational parameters chosen are, however, confidential. Threat scenarios were then derived in integrated working groups putting this threat sensor situation into a relevant context.

There is no clear-cut distinction made by the respondents between stakeholder requirements definition and requirements analysis at different levels in the process described. Nor is this to be expected, since the government and industry participants worked so tightly together. From the description of the system requirements analysis process in the next subsection, however, it is understood that there is a need for the input of mission scenarios.

\subsection{The system requirements analysis}

"The purpose of the Requirements Analysis Process is to transform the stakeholder requirement driven view of desired services into a technical view of a required product that could deliver those services...It results in measurable system requirements that specify, from the supplier's perspective, what characteristics it is to possess and with what magnitude in order to satisfy stakeholder requirements $, 6(\mathrm{p} 69), 13$

The results of the SAT/Mark working group on requirements specification were documented in FMV Guidelines for requirements on signature management ${ }^{10}$ (Restricted). One of the respondents, however, highlighted their most important positions on the requirements process.

Firstly, LOT and signature management are needed in order for a platform to survive and accomplish its military task. This led to a conclusion that the advance to contact is the dimensioning phase of a mission ("not standing still hiding in a forest") since this phase is where the vehicle signature properties are most critical.

Consequently, requirements analysis has to start with analyzing tactically correct movement in typical situations because this dictates the critical signature level. The working group concluded that it is very important to decide upon dimensioning typical situations, where signature management is important to the mission. Discussing signature from a general viewpoint is futile. Hence, what are really needed from the customer when doing requirements analysis are 
relevant scenarios. From these typical situations, the sensor threat, the physical environment, the modus operandi, the vehicle operating conditions and the capabilities needed can be derived.

Another important aspect identified was the probability of the sensor threat occurring in the scenarios. What sensors are volume threats, i.e. mass deployed sensors, and what sensors are exclusive threats or advanced sensors not yet common in the theatre? There might be a new exclusive sensor someone has recently heard of, but should that be allowed to be a factor in the design of the vehicle signature, the respondent asks rhetorically. Instead, the working group arrived at the conclusion that the volume threat should be prioritized. From a technical perspective, one can always say that, if this or that sensor is available in theater, it will, or will not, be able to see objects. On the other hand, the consequences for the system of interest have to be analyzed. And if a new threat sensor appears somehow, one will have to adapt, the respondent concludes. This was also one of the lessons reported by Olsson et al. ${ }^{1}$

Next the analyst has to consider in what mode the threat sensors are working when detecting the combat vehicle. Some sensors do have an impressive resolution, but perhaps only if working in a very narrow field of view. Maybe the scanning mode instead puts moderate requirements on signature management. If the platform survives the first seconds of the threat sensor scanning its surrounding terrain, before it goes into high resolution mode, then the time to detection is extended considerably. Different sensor types were discussed and tabulated by the working group, with typical resolutions, fields of view and working distances. The respondent gave an example when discussing end phase corrected ammunition. Such a weapon is probably not launched unless the target is found using another sensor, and what is the field of view needed for that?

Another important experience of the requirements working group was that signature management requirements are often overstated. It is sufficient to state dimensioning requirements, the respondent states. The working group concluded that solution space should be explored by using simulations in order to find the relevant requirements. However, back then there were less sophisticated simulation tools than there are today, the respondent explains.

Since the signature management requirements for SEP were largely adopted from the demonstrator program (with some exceptions to be noted later), the requirements analysis process, - starting with the identification of dimensioning typical situations, described above and developed during the concept stage - appears to have been validated in the SEP program. It should be noted, however, that the input wanted from the military organization appears to be a rather dense scenario, comprising descriptions of anticipated missions, own tactics and procedures, anticipated physical environments and anticipated adversaries. A comparison with concepts of operations or a further description of the design of such a scenario would be useful.

The dimensioning situation was found to be the advance to contact phase. This of course supports interest in controllable vehicle signatures and emphasizes the need to be able to state relevant and measurable requirements of temporal contrasts in all sensor wavelength bands. Increasing interest in controllability was anticipated by several respondents, especially for exclusive assets, but no suggestions were made regarding metrics or how to handle this category of requirements. Another respondent raised serious doubts about controllability ever becoming a reality. It is technically difficult and its utility on a dirty battlefield would be limited he argues. Controllability results from the demonstrator project were published in $2003^{14}$.

Furthermore, the challenge is not any difficulty in getting the scenario information needed to initiate the process from end-users. However, transforming the mission scenarios or user needs into technical system requirements of the signature does remain a challenge, the respondents admit. In addition, and unfortunately, the Swedish standardization work during the SAT/Mark program did not finish. The respondents' observations in many cases are that this transformation has been avoided by, instead, stating technically oriented requirements, such as what color paint to use or the temperature difference to ambient air instead of background, leading to uncertainty about whether or not the requirements reflect real conditions. 
One respondent described an ambition, which arose during the work, to develop a causal chain of measures and methods between what can be measured in the laboratory and what the operator in the field can experience. The approach described aims to derive verifiable signature requirements from contrast measures on pictures of views seen by the threat sensor operators. Promising results are to be published. This has the obvious benefit of making requirements independent of sensor wavelength spectra.

The linking of tactically relevant parameters to verifiable, measurable, technical system requirements is a major issue. The authors' view of the emerging system model for evaluation of signature management in combat vehicles can be seen in Figure 4. The model is transferred from the aircraft combat survivability discipline ${ }^{5,15}$ and modified to the land theater to capture issues highlighted by the respondents. The purpose is to illustrate the linkage between the performance of a combat vehicle, threat sensor systems and mission needs. The output measures of performance at one system level are response functions of lower system level inputs and are themselves input to higher system levels. The capability system level has been added because the manner in which a combat vehicle is used in theater greatly affects performance measures, such as 'Time to detection' suggested by respondents.

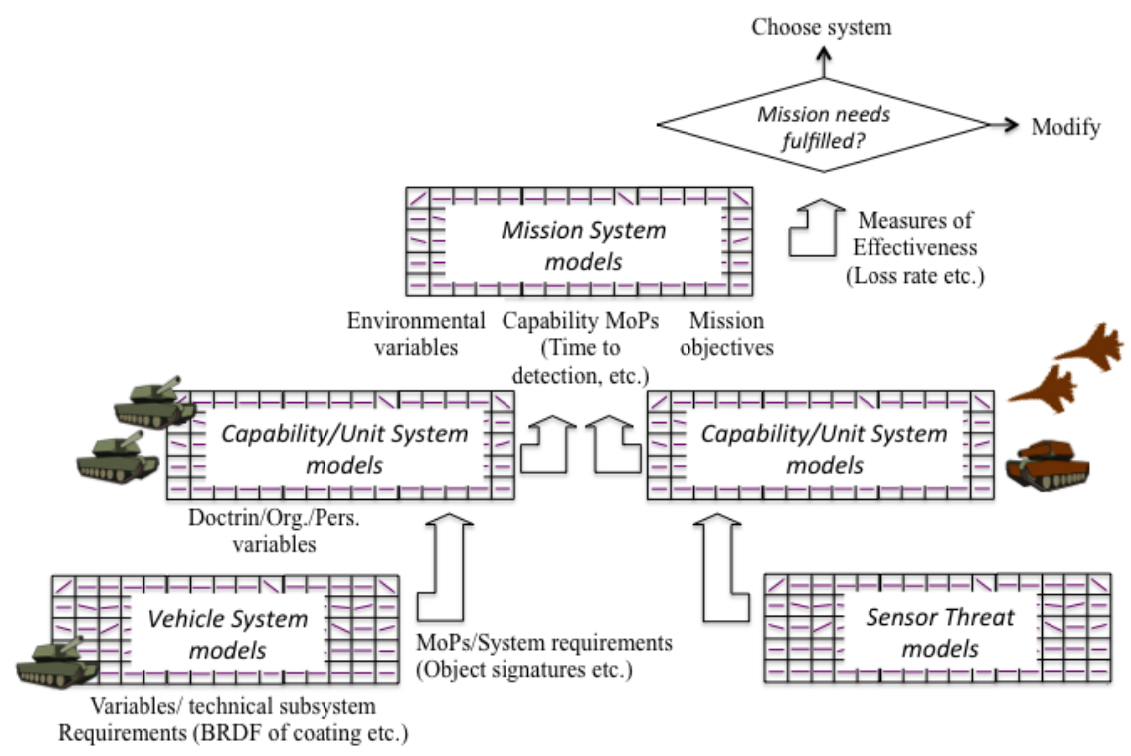

Figure 4. An illustration of the systems model supporting analysis in the development of a combat vehicle with signature requirements. The output measures of performance at one system level are response functions of lower system level inputs and are themselves input to higher system levels. The illustration is inspired by the work of Soban and Mavris (2002) $)^{15}$.

It goes without saying that, at the vehicle system level, the general measure of performance is object signature. But how should it be measured? The SAT/Mark working group decided on a definition of signature that includes the objects interaction with the environment, signature is the contrast between object and background making the object detectable in any wavelength and characteristic ${ }^{9}$. A radar cross section is consequently not a signature but a property of the object. One of the respondents exemplifies: For a SAR sensor the signature is the object and its shadow, for an IR sensor hot air from the object affects the background and in the visible spectrum dirt tracks in the background caused by the vehicle should be included. Hence, a requirement at the signature level must be accompanied by a set of situational parameters. 
It is also evident that signature should be measured with a palette of contrast measures - with whichever has the greatest impact on measures of effectiveness at the top. One of the lessons, presented in 2002 by Olsson et al, suggests dividing these contrast measures into three categories because of their similarities across wavelength bands: spatial, spectral and temporal, and to take into account whether or not the target is detected by a passive or an active sensor. However, as recently stated by Åkerlind et $\mathrm{al}^{16}$, there seems to be no generally accepted set of contrast measures to fill the matrix completely. From the authors' experience there is a lot of research in some of the matrix elements, with several measures to choose between, while in others there is a lack of research. In addition, the work to form a Swedish requirement standard did not finish. However, given the development towards multinational procurement projects, standardization should continue in a multinational context. One of the respondents describes a recent EDA initiative stating generic requirements in an effort to create action preparedness for future joint projects.

An issue touched upon above is the choice of situation parameter sets needed to form signature requirements. The challenge, when compared to other attributes is the statistical nature of signature, i.e. the dependence on physical environment. One of the consequences described by one respondent is the necessity to measure the signature of an object on several occasions and in different environmental conditions. This is necessary in order to find the extremes and hence be able to interpolate other parameter sets, and thus be able to assess the signature under any conditions, the respondent states. Another consequence is that it is sufficient to state requirements for dimensioning parameter sets to avoid overstating. Otherwise, this could, of course, lead to unnecessary testing, possibly contradictory requirements and other project risks. Hence, at some point after the identification of typical situations, it is necessary to define the environmental parameters under which signature requirements should be stated. A reasonable conclusion is that finding arbitrary points in solution space requires sophisticated systems modeling in both cases.

The 'Time to detection' measure of performance was often found to be more important than detection range as the tactical parameter from which to derive vehicle system requirements. In Swedish mission scenarios the range is usually much longer than the line of sight available on the ground, one respondent explains. If the maximum range is $5 \mathrm{~km}$ but the target vehicle is standing one km away from the sensor, there is a risk of being detected the whole time. But will the sensor react to the target? That is the important question. Reducing detection range from five to one kilometer using signature management technology is of no use. In this situation it is all about contrasts and the background statistics, the respondent concludes. Another argument put forward was that it is impossible to do a relevant static comparison between the ranges of X-band radar and an optical high-resolution sensor. The X-band sensor is a quick scanning sensor, while the optical sensor has a limited field of view. Hence, it is important to formulate requirements for both detection range and time to detection, in scenarios covering the threat sensor suites' different modes of operation, another respondent concludes. A third respondent advocates 'Time to classification' as an important parameter since, in sophisticated sensor threat scenarios, it is difficult to delay detection at short range. The military operator will probably not shoot immediately on detecting a target, unless there is additional information, he argues. That means the vehicle firing will have to get closer to the target, or wait until the target comes closer, in order for the operator to have the required resolution to classify or identify. Detection is about contrast between target and background, but classification is also partly dependent on contrasts within the target, he ends, and implies that this is somewhat easier to control with signature management. In Olsson et al, 2002, it is stated that relevant tactical parameters have to be derived from the context in each case. ${ }^{1}$

Discussing the linking of system requirements to tactically relevant parameters, difficult as it is, does not, however, capture the full complexity of survivability engineering. Returning to the lessons from survivability engineering in the combat aircraft discipline (See Section 2), Ball stresses the need to measure mission outcomes at the next system level (see measures of effectiveness at the top level in Figure 4), or even campaign outcomes, in order to see the real military value of signature reduction features in platforms ${ }^{5}$. Otherwise one might miss penalties in the platforms lethality or availability when doing the evaluation, or one might underestimate the real value of low signature and invest in other attributes instead. The issue is touched upon in Olsson et al, but is limited to comparing different survivability 
enhancement techniques. The validation results from the SAT/Mark demonstrator are, however, classified; hence it is not clear to the authors what effects signature trade-offs had on the vehicle meeting mission needs.

\subsection{The architectural design process and trade offs}

"The purpose of the Architectural Design Process is to synthesize a solution that satisfies system requirements...It identifies and explores one or more implementation strategies at a level of detail consistent with the system's technical and commercial requirements and risks. From this, an architectural design solution is defined in terms of the requirements for the set of system elements from which the system is configured..."

In their paper Olsson et al describe their system approach to design. ${ }^{1}$ Given that signature is an attribute of the whole system of interest and that, at system level, it is difficult to predict the exact effect of signature enhancement measures to subsystems, the design process has to be iterative. The first iteration of allocating system requirements to subsystems is done by breaking them down into subsystem guidelines and subsystem requirements, but within the ambition for the overall design to meet all system level requirements. After evaluation the allocation is adjusted for the second iteration etc. until system requirements are met.

This approach to design seems to have been validated during the SEP development. One of the respondents illustrated the applied approach, and the special implications of signature management design, with an example from the SEP development - how to get rid of excess heat. The system signature requirement originated from a certain acceptable level of heat emission, the respondent explained. Firstly, the system designer distributes permissible heat emission evenly to all surfaces of the vehicle. However, it is then realized that a larger part of the budget has to be allocated to the designer of the exhaust subsystem, who is struggling to conceal the flow of one cubic meter of hot air per second. Now the system designer can no longer formulate requirements from a signature perspective; they would not make sense. The system signature requirements have to be transformed into concrete subsystem design guidelines. In this particular case Hägglunds used counter flows of air, in a design later patented. However, they could not be sure of meeting the system requirement until a prototype of the complete system was integrated and evaluated. In fact the process is recursive and there were several subsystem levels with iterations including tests at every level, the respondent continues. For instance, the exhaust system was first validated in a bench configuration at subsystem level. In contrast to signature requirements, the maximum weight requirement is easy to break down into subsystem requirements. If a vehicle's maximum weight is 25 tons, the transmission can only weigh three, the respondent exemplifies.

Another lesson identified at an early stage was the "work from the inside out" approach to design. The design organization should strive to meet signature requirements as early as possible during basic construction. The priority order is dictated by the consequences of correcting a poor design later on: Radar, TIR and VIS (NIR-VIS-UV). Inappropriately designed inner corners are for example difficult to redesign later. Furthermore, if the infrared signature is considered early, it will be easier to deal with the excess signature using coatings or mobile camouflage systems (MCS) etc. It might be possible to go a long way with an MCS, but with poor basic construction, the design of the add-on becomes more complicated, and hence there are penalties in life cycle cost or other performance attributes of the combat vehicle system. This lesson is also in complete accordance with the first point made by Ball (See section 2).

The purpose of the low observable technology demonstrator was to push stealth performance in a combat vehicle; hence, when stating the signature levels for a supposedly operational vehicle like the SEP, there has to be some trade-offs. For instance, the radar requirements, when compared with the demonstrator, were lowered for two reasons. Firstly, the handles for hatches etc. on the demonstrator were designed with low radar signature in mind - forcing the operator to remove his or her gloves. Secondly, lowering the requirements also made it possible to use traditional components. Hence, for SEP, radar signature was traded for usability and for ease of production. Given the discussion earlier about balancing requirements by taking the probability of different sensor threats into account, the trade-off decision was likely based on systems modeling and an assessment of relatively low probability of radar occurring in theaters at the time, like 
e.g. Afghanistan. However, slight reductions in both mobility (to benefit from skirts) and situational awareness (fewer electro optical sights to reduce reflections) were accepted, no doubt originating from an assessment that there are many more threat sensors in TIR and VIS.

Another important lesson from the design organization is that a system designer can achieve a lot in signature reduction using standard components - if signature management is allowed to influence design from the beginning. There is a greater effort in the systems engineering involved, but the unit price need not be much higher than it otherwise might, one respondent concludes. This conclusion is from an industry representative. A statement from the government side, however, supports the conclusion, since the respondent cannot remember any trade-offs to cost originating from signature requirements. Validating the statement with a financial analysis is beyond the scope of this study.

\subsection{Integration, verification and validation}

"The purpose of the Integration Process is to assemble a system that is consistent with the architectural design..." $6(\mathrm{p} 118), 13$

"The purpose of the Verification Process is to confirm that the specified design requirements are fulfilled by the system..., 6(p123),13

"The purpose of the Validation Process is to provide objective evidence that the services provided by a system when in use comply with stakeholders' requirements, achieving its intended use in its intended operational environment." $6(\mathrm{p} 133), 13$

The respondents state that there was no system level verification and validation campaign for the SEP vehicle because the project was terminated beforehand. There were, however, some technical simulations to validate system performance of the SAT/Mark demonstrator. Although when it comes to validation methods the SAT/Mark project was incomplete, one respondent explains. Validation was based on discussions with the end user. The need to develop methods and tools was identified. There was also some statistical evaluation of how the target fits into backgrounds. Some FOI reports have been published on the subject, but these methods have not been validated and approved to the respondent's knowledge.

For the SAT/Mark demonstrator a new surface with "extraordinary" BRDF (bidirectional reflection distribution function) properties was developed and applied to the vehicle. The surface was about $5 \mathrm{~mm}$ thick consisting of glossy cones. However, because the cones were directed in so many directions, they mirrored each other resulting in a very deep color, one respondent explains. This coating was to maintain color from all viewing angles. The validation using a new BRDF instrument was reported by Olsson et al. ${ }^{1}$ It was, however, difficult to make the surface stick to the demonstrator plates and it has not entered production.

Measuring the BRDF value of a large enough surface sample is one method proposed for linking sub-system requirements to what a threat sensor operator experiences in the field, as touched upon earlier (Olsson et al, 2002). The problem is that requirements are usually stated for the raw material, the flat stock, and not the punched and finished product, a respondent explains. Hence, when verifying these requirements, you are not really evaluating the product seen in the field by a threat sensor and an observer. The problem originates from difficulties in obtaining relevant measurements, the respondent concludes. The authors agree. The dispersive properties of a surface have a huge influence on detectability. Full dispersion for all incident and reflection angles and all wavelengths is described by the BRDF, but, to the authors' knowledge, this can only be measured at a few sites in Europe. FOI (Linköping) and Saab Barracuda (Gamleby), whilst having some limitations, are two of them.

When asked for their view on future challenges, one of the respondents brought up an interesting challenge using modeling and simulation techniques in the verification and validation of signatures. One problem emerging, he said, is that signatures are now becoming very low, which is actually a problem in modeling and simulation. Detection is a subtler phenomenon to simulate than before, because it has to be based, perhaps, on a glint from the target showing a 
high signature aspect angle for a brief moment. Is it possible to identify and track that target, the respondent asks rhetorically, and concludes that the system effectiveness measure will have to be more complex, and the simulations will require more detailed data to be relevant.

\subsection{The study method}

Two facts raise questions about the validity and reliability of the results: Firstly, the SEP has not (yet) been built, verified and validated; so, how can we be certain the requirements specification is well balanced? Secondly, important findings and results, such as signature measurements, are classified. However, this case is unique and scrutiny is well justified. Reliability is helped by having had the opportunity to interview key actors in the case, and by the fact that they are very experienced and still active in the field. Tendencies do not seem to be a severe problem since the respondents are reasonably coherent.

\section{CONCLUSIONS- THE SYSTEMS APPROACH REVISITED}

The Systems approach to development of stealth on the ground, proposed to SPIE in 2002, was summarized in a shortlist of lessons on modeling and analysis. The shortlist was then brought in as the background to an interview study, from a signature management and systems engineering perspective, on the development of the Swedish Armored Multipurpose Vehicle, SEP. The lessons reported earlier were found to be at least partly validated during the SEP development. No contradictory findings are reported. The complete list of earlier lessons(*), and those identified from the study, are as follows (in logical, not priority, order):

Success factors:

1. *Functional analysis starts with mission needs and weapons/sensor threats as input, to determine the relative importance of stealth compared to other means of survivability. The overall survivability goal is considered under all the different operating modes of the vehicle system. Different sets of system functions form alternative concepts to be evaluated using system modeling or war gaming. ${ }^{1(\mathrm{pp} 3-4)}$

2. *A model based on weighted probabilities of sensor occurrence is used to derive signature level requirements for corresponding wavelength domains. The boundary condition is set to specify a system with a balance between corresponding signatures in as many backgrounds as possible. ${ }^{1(\mathrm{pp} 3,5)}$

3. *The definition of a system model comprises vehicle system models, and sensor threat models for relevant signature

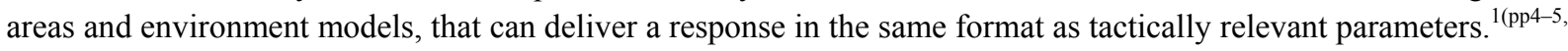
7)

4. *A UV/VIS/NIR sensor model needs at least the following input variables, in addition to background and foreground, to produce a usable response parameter: object size, object shape, object contours, object shadows, atmospheric blurring and noise, atmospheric wavelength dependent attenuation, color (value and gloss) and specular properties. $^{1\left(\mathrm{pp}^{7-9)}\right.}$

5. The signature definition (see section 4.2). It is the basis for defining contrast measures, i.e. the vehicle system measures of performance. There is no international standard.

6. An integrated team approach is an important enabler in the systems engineering of any complex system, but the results indicate that this is especially important in the analysis and design of a stealthy vehicle signature, it being a systems attribute. In an integrated project team, at the concept stage, there should be representatives from the endusers, the procurement agency, scientists and, if any commercial constraints allow, industry experts. Creating an atmosphere of openness and cooperation is essential. 
7. *The allocation of system requirements to sub-systems starts from an established conceptual system design, thereby assuming set system signatures, and defining sub-system requirements in the form of design guidelines. Design guidelines typically include requirements for specific functions, material properties, dimensions, shape or surface properties etc. Then the system signature should be evaluated, preferably using system modeling, and the procedure reiterated until system requirements are met. This procedure is necessary because of the systemic nature of the signature attribute, i.e. because signature requirements have no meaning at subsystem level. ${ }^{1(\mathrm{p} 6)}$

8. When designing a stealthy combat vehicle, (electromagnetic) signature requirements should be considered as early as possible in basic construction to optimize performance and minimize cost. The order of priority should be radar, TIR, VIS (incl. UV and NIR).

9. *Categorization of system requirements should be detection-process-oriented, rather than by sensor type. Methods for analysis and requirements specification tend to be the same, regardless of wavelength. Use of a $3 \times 4$ matrix structure with spatial, spectral and temporal contrast on the vertical axis and with unresolved/resolved targets, using either passive or active sensors, on the horizontal axis is suggested. ${ }^{1(\mathrm{pp} 5-6)}$

Observations:

10. Time to classification is perhaps equally interesting as time to detection as the capability measure of performance conveying military needs to the vehicle system level.

11. There is a need for an agreed standard on preferred contrast measures in all elements of the $3 \times 4$ matrix above, see item nine.

12. *In VIS a surface optical response should be modeled, or specified, using its BRDF value. Results show that illumination angle and viewing angle have a greater effect on contrast than choice of color. The BRDF value incorporates this effect. ${ }^{1(\mathrm{pp} 9-10)}$

13. Stating requirements for dimensioning situation parameters is sufficient. Finding these using systems modeling is a research area.

14. If signature requirements are allowed to influence the procurement process from the beginning, stealth is not expensive.

15. In a well working integrated team there is a risk of being tempted not to produce thorough documentation, thereby impeding the traceability of requirements.

Work needed:

16. The procurer's stakeholder requirements analysis starts with the analysis of mission scenarios established by the military user organization. The 'scenario' concept in this context needs elaboration.

17. The transformation of mission scenarios into measurable, technical systems requirements still needs clarification. An approach establishing linkage between tactically relevant parameters, such as time to detection, through relevant and measurable contrasts in the picture seen by the sensor system operator is suggested.

18. Establishing the linkage from vehicle system measures of performance to tactically relevant parameters is, however, not enough to do trade-offs between signature reduction features and other features linked to lethality or availability. Establishing a mission system model, linking those tactically relevant parameters to mission objective measures of performance is required. 
19. The modeling, analysis and formulating of requirements for controllable signatures needs special attention. The dynamic contrast of a vehicle moving against a background should be studied further.

20. There is a need to define measurable contrast measures for those matrix elements lacking candidates, see item nine.

In conclusion the items above are candidate guidelines for a suggested approach to the system engineering of stealthy vehicles. However, the method has not been validated by being applied to a completed procurement case. This should be done as part of a future major procurement project. The 'Systems approach' to stealth on the ground, and the projects where it was born, are well worth revisiting!

\section{AKNOWLEDGMENTS}

This work was financially supported by the Swedish Defence University. The authors would like to thank Mr Stephen Henly for language editing the manuscript, Mr Martin Bang and Mr Anders Grop for valuable inputs and of course the respondents for their generosity with personal time and for sharing their valuable experience.

\section{REFERENCES}

\subsection{Publications}

[1] Olsson Ö, Karisson L, Lindwall P, Dickman O, Grop A., "A Systems Approach to Stealth on Ground ; SAT/Mark Technology Demonstrator", Proc. SPIE 4718,1-11 (2002).

[2] Lindström R. O., "SEP: Multirole Armoured Platform", Swedish J Mil. Tech., - (1999).

[3] Försvarsmakten, "Materielförsörjningsstrategin 2007", HKV 23 251:61994, 2007-02-02, 1- (2007)

[4] Lindström R. O., "När AWV blev AMV", Pansar (2), 4-9 (2008).

[5] Ball R. E., [The Fundamentals of Aircraft Combat Survivability Analysis and Design], 2nd ed., Reston, VA: AIAA Education, (2003).

[6] Haskins C, Forsberg K, Krueger M, Walden D, Hamelin D., [INCOSE - SE Handbook], 3.2 ed., INCOSE, (2011).

[7] FOA, "SAT/Mark (MOC) Slutrapport SG modellering", FOA-R--98-00883-615, 1- (1998).

[8] FOA, "SAT/mark (HKC) Slutrapport studiegrupp Krav", FOA-R--98-00874-615, 1- (1998).

[9] Bohman L., [Handbook on signature management technology for ground] (swedish), FOI, (2003).

[10] FMV, "FMV Guidelines for requirements on signature management", FMV 29156/2006 H/R, Issue 13, (2006).

[11] Försvarsmakten, "MS 136 SEP - Reviderad Preliminär Taktisk Teknisk Ekonomisk Målsättning (RPTTEM) för Splitterskyddad EnhetsPlattform (SEP)", HKV H 35 129:80713, 2006-06-12, (2006).

[12] Lindström R. O., Nilsson L, Tapper K, "Slutapport MS 136 SEP", FMV 34719/2009, 2009-11-25, (2009).

[13] ISO/IEC, "ISO/IEC 15288:2008 - Systems and software engineering - System life cycle processes", (2008).

[14] Westin J, Olsson O, Pettersson L, Lindwall P, Grop A., "Active control of infrared signature: system implementation in a ground vehicle", Proc. SPIE. Vol. 5075, 39-48 (2003).

[15] Soban D, Mavris D., "Formulation of a Methodology for the Probabilistic Assessment of System Effectiveness", AIAA Strategic and Tactical Missile System s Conference. Monterey, CA: AIAA, 1-12 (2000).

[16] Åkerlind C, Hallberg T, Kariis H, Fagerström J., "Evaluation criteria for spectral design of camouflage", Proc. SPIE 9653, (2015).

\subsection{Interviews}

Lars Bohman, MSc, currently head of the department for radar systems at FOI, in a semi-structured interview on the 23rd of April 2015 at FOI in Linköping. Lars Bohman was interviewed in his capacity as senior scientist representing FOI in several working groups during the SAT/Mark program. He was chairman of the sensor threat assessment-working group and of the working group on how to state requirements for signature management. 
Ola Dickman, $\mathrm{PhD}$, currently the project manager for FMV signature management R\&D, semi-structured interview on the 25th of February 2015 at FMV. Ola Dickman was interviewed in his capacity as the project manager of the SAT/Mark demonstrator project and as project manager of the FMV R\&D group on signature management. He was also project manager for the procurement of mobile camouflage systems for some of the combat vehicles during the period of interest and also for R\&D projects directed to Barracuda for technological development.

Lars Karlsson, BA, currently consultant involved in evaluation of Saab Barracuda AB products, semi-structured interview on the 8th of April 2015 at Saab Barracuda in Gamleby. Lars Karlsson was interviewed in his capacity as the senior systems engineer representing Saab Barracuda in the SAT/Mark project.

Rickard O. Lindström, MSc, currently strategic specialist for combat vehicles at FMV and POC for international cooperation, semi-structured interview on the 25th of March 2015 at FMV in Stockholm. Rickard O. Lindström was interviewed in his capacity as the FMV project manager of the SEP project from start to finish, 15 years in total. During the SEP development phase he was also program manager at FMV with overall product responsibility.

Örjan Olsson, PhD, currently vice president of BAE Systems Hägglunds, semi-structured interview on the 6th of March 2015 at BAE Systems Stockholm office. Örjan Olsson was interviewed in his capacities as specialist in signature management, during the technology demonstrator project, then specialist and systems engineer during the SEP development project and, finally, project director with overall responsibility for the product.

Kenneth Tapper, LTC (Retd), currently consultant involved in another SwAF development project, semi-structured interview on the 4th of May 2015 at FMV. In 1996 Kenneth Tapper was interviewed in his capacities as system manager in SwAF for mechanized infantry, until 1999, then the SwAF manager of the SEP project, head of the R\&D committee for combat vehicles and also the Swedish head representative in a project with the UK on a tentative collaborative procurement of combat vehicles. 